\title{
On the Origin of the Spiral Morphology in the Elias 2-27 Circumstellar Disk
}

\author{
Farzana Meru, Attila Juhász, John D. Ilee, Cathie J. Clarke, Giovanni P. Rosotti, and Richard A. Booth \\ Institute of Astronomy, University of Cambridge, Madingley Road, Cambridge CB3 OHA, UK \\ farzana.meru@ast.cam.ac.uk, juhasz@ast.cam.ac.uk, jdilee@ ast.cam.ac.uk \\ Received 2017 February 2; revised 2017 March 8; accepted 2017 March 14; published 2017 April 19
}

\begin{abstract}
The young star Elias 2-27 has recently been observed to posses a massive circumstellar disk with two prominent large-scale spiral arms. In this Letter, we perform three-dimensional Smoothed Particle Hydrodynamics simulations, radiative transfer modeling, synthetic ALMA imaging, and an unsharped masking technique to explore three possibilities for the origin of the observed structures - an undetected companion either internal or external to the spirals, and a self-gravitating disk. We find that a gravitationally unstable disk and a disk with an external companion can produce morphology that is consistent with the observations. In addition, for the latter, we find that the companion could be a relatively massive planetary-mass companion $\left(\lesssim 10-13 M_{\text {Jup }}\right)$ and located at large radial distances (between $\approx 300-700 \mathrm{au}$ ). We therefore suggest that Elias $2-27$ may be one of the first detections of a disk undergoing gravitational instabilities, or a disk that has recently undergone fragmentation to produce a massive companion.
\end{abstract}

Key words: hydrodynamics - planet-disk interactions - protoplanetary disks - radiative transfer - stars: individual (Elias 2-27) - stars: pre-main sequence

\section{Introduction}

With the advent of the Atacama Large Millimeter/ submillimeter Array (ALMA), it has for the first time become possible to spatially resolve, and thus directly observe, the midplane structure of protoplanetary disks where planet formation processes occur. Such an extreme increase in observational capability has given rise to several surprising results, examples of which include the symmetric ring structures in HL Tau (ALMA Partnership et al. 2015), TW Hydrae (Andrews et al. 2016) and HD 163296 (Isella et al. 2016), the horseshoe-shaped dust traps in HD 142527 (Casassus et al. 2013), and the birth of a ternary system via disk fragmentation in L1448 IRS3B (Tobin et al. 2016). Observations such as these are extremely powerful, as sub-structure within protoplanetary disks can be a signpost of dynamical or chemical effects occurring within the star-disk system. Therefore, a proper understanding of their cause is essential to determine which of these processes are important during the star and planet formation process.

A recent example of such a spatially resolved observation was presented by Pérez et al. (2016) in which Elias 2-27 was targeted with ALMA. Elias 2-27 is a low-mass young star $\left(M_{\star}=0.5-0.6 M_{\odot}, t_{\text {age }} \sim 1 \mathrm{Myr} ;\right.$ Luhman \& Rieke 1999; Natta et al. 2006). Based on its spectral energy distribution (SED), the system is thought to belong to the Class II phase (Andrews et al. 2009; Evans et al. 2009), yet at the same time, observations have suggested an unusually large disk mass, ranging from 0.04 to $0.14 M_{\odot}$ (Andrews et al. 2009; Isella et al. 2009; Ricci et al. 2010).

The Elias 2-27 disk posseses two large-scale symmetric spiral arms (Figure 1, right). Additionally, when the raw ALMA observations are processed with an unsharp masking filter, two dark crescents interior to the spirals and a bright inner ellipse are revealed (Figure 1, left). The origin of these is unclear. With such a large disk-to-star-mass ratio, could the disk be self-gravitating? Or, could an as-yet-undetected companion be causing these features via dynamical interactions?

In this Letter, we describe the results of hydrodynamical and radiative transfer modeling of the Elias 2-27 system. We produce synthetic ALMA observations to explore three possibilities that may give rise to the observed features-a companion internal to the spirals, a companion external to the spirals, or gravitational instabilities operating within the disk.

\section{Methodology \\ 2.1. Hydrodynamics}

Our simulations are performed using a three-dimensional Smoothed Particle Hydrodynamics code (SPH) which includes the heating due to work done and the radiative transfer of energy in the flux-limited diffusion limit (Whitehouse et al. 2005; Whitehouse \& Bate 2006). A detailed description of the code can be found in Meru (2015); however, for this work we employ two differences. First, boundary particles are located at every time step, allowing the vertical location between the optically thick and thin regions to be regularly reevaluated, leading to more accurate boundary temperatures. Second, we employ the Morris \& Monaghan (1997) artificial viscosity with the $\mathrm{SPH}$ parameter, $\alpha_{\mathrm{SPH}}$, varying between 0.1 and 1.0 and $\beta_{\mathrm{SPH}}=2 \alpha_{\mathrm{SPH}}$, to model shocks within the disk.

We perform 72 hydrodynamical simulations varying a number of disk properties as well as orbit properties for the companion simulations. We first describe our reference disk setup: we model a $0.5 M_{\odot}$ star surrounded by a disk whose temperature follows

$$
T(R)=13.4 \mathrm{~K}\left(\frac{R}{200 \mathrm{au}}\right)^{-q} .
$$

The disk surface density follows

$$
\Sigma(R)=\Sigma_{\mathrm{c}}\left(\frac{R}{R_{\mathrm{c}}}\right)^{-p} \exp \left(-\left(\frac{R}{R_{\mathrm{c}}}\right)^{2-p}\right)
$$



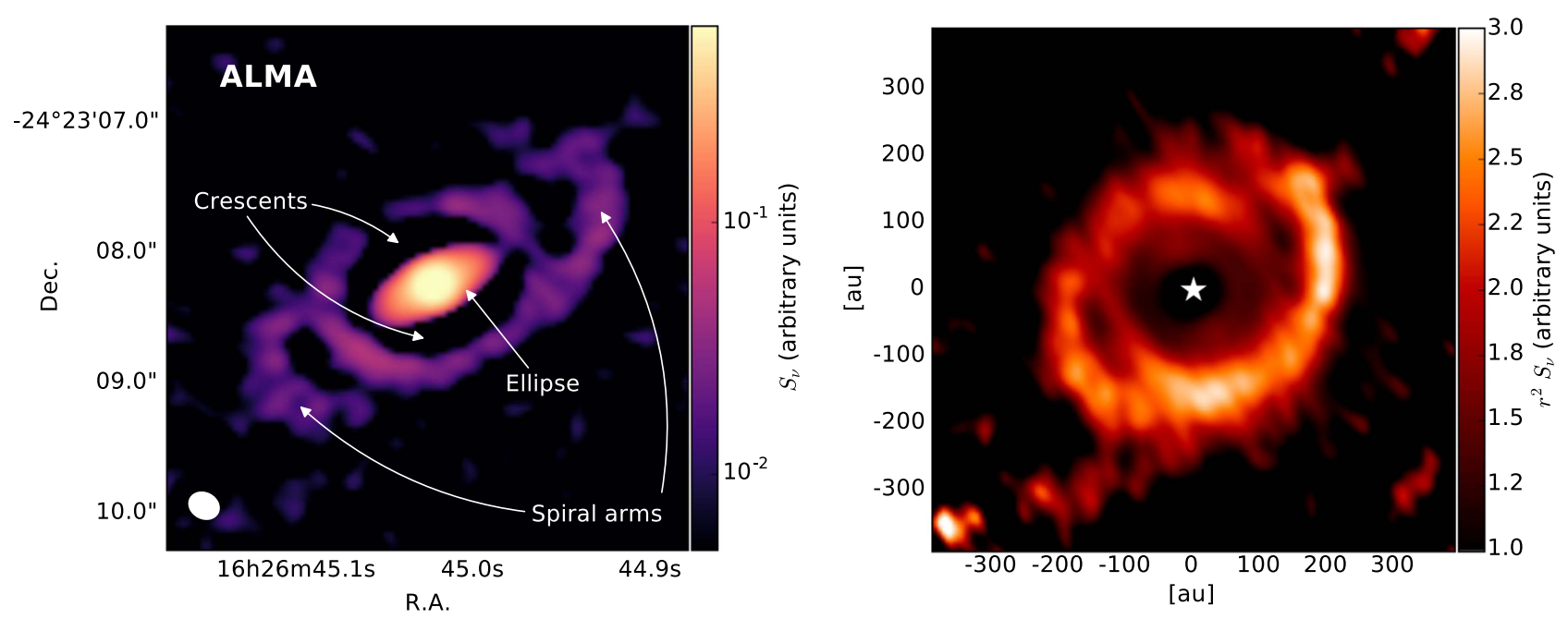

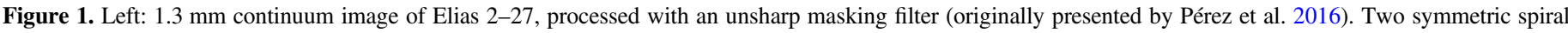

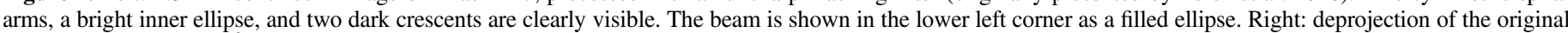

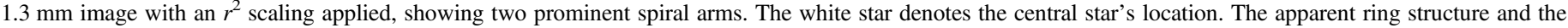
central ellipse in the left image are artifacts of the unsharp masking.

where $\Sigma_{\mathrm{c}}$ is the surface mass density of gas at the cutoff radius, $R_{\mathrm{c}}$. We model this disk between $R_{\mathrm{in}}=10$ au and $R_{\text {out }}=400 \mathrm{au}$ using the parameters determined by Pérez et al. (2016), namely, $\Sigma_{\mathrm{c}}=5 \mathrm{~g} \mathrm{~cm}^{-2}, R_{\mathrm{c}}=200 \mathrm{au}, q=0.45$, and $p=0.7$.

We then perform a suite of simulations with a wide range of parameters to test the internal companion, external companion, and gravitational instability hypotheses. Due to the computational expense of each simulation, our aim is not to fit the exact parameters, but to test whether each hypothesis can reproduce the observed morphology. We vary the disk mass, surface mass density profile, temperature profile, cutoff radius, and outer disk radius. We also model pure power surface density profiles, i.e., without the exponential term in Equation (2), so that, once evolved, the disk has a much steeper profile in the outer regions. In addition, for the companion simulations, we vary the companion mass, the pericenter distance, eccentricity, and inclination.

Each disk is modeled using 250,000 SPH gas particles. The ratio of the smoothing length to the disk scale-height is $<0.5$ outside 40-60 au, giving sufficient resolution to probe dynamical effects on the disk. The disks are modeled with radiation hydrodynamics using flux-limited diffusion, and the surface temperature (representing irradiation from the central star) is held at the profile given by Equation (1). For the parameters studied here, the disk is optically thick to stellar irradiation and remains vertically isothermal at the boundary temperature beyond a radius of 20 and 30 au in the companion and self-gravitating disk simulations, respectively. This implies that the thermodynamics are mainly set by external irradiation.

We assume that the gas and dust are well mixed. For our self-gravitating simulations the Stokes numbers of millimeter particles are $\mathcal{O}(0.01)$, and thus dust trapping in the spirals is expected to be marginal (Shi \& Chiang 2014; Booth \& Clarke 2016). In our simulations with a companion, the Stokes numbers are $\mathcal{O}(0.1)$. For a spiral to trap dust, however, it is also necessary that the timescale for it to concentrate toward the pressure maximum (Clarke \& Lodato 2009) is less than the crossing timescale of a spiral feature. Although this condition is readily met in the case of self-gravitating disks where the spirals nearly co-rotate with the Keplerian flow, this is not true in the case of a planet-generated spiral that co-rotates with the planet (e.g., Paardekooper \& Mellema 2006; Zhu et al. 2012; Birnstiel et al. 2013).

\subsection{Radiative Transfer and Synthetic Imaging}

To calculate synthetic observations of our models, we use the $3 \mathrm{D}$ radiative transfer code RADMC-3D. ${ }^{1}$ The dust opacity is calculated from the optical constants for astronomical silicates (Weingartner \& Draine 2001) using Mie-theory, assuming a power-law grain size distribution between 0.005 and $1000 \mu \mathrm{m}$ with a power exponent of -3.5 . Outside 80 au we obtain the dust temperature and density on the spherical mesh by interpolating the gas density and temperature from the hydrodynamic simulations using an SPH interpolation with a cubic spline smoothing kernel (Monaghan 1992) and assume a uniform gas-to-dust ratio of 100. Due to the accretion of SPH particles on the central star, we extrapolate the density inwards of 80 au using a radial power-law with an exponent chosen to give a smooth transition from the results of the hydrodynamic modeling. We assume a vertical Gaussian density distribution, whose scale-height is calculated from the temperature given by Equation (1). The dust and gas temperatures are assumed to be identical. Using these temperatures and densities on a spherical mesh, we calculate images at $\lambda=1.3 \mathrm{~mm}$ using the raytracer in RADMC-3D.

Synthetic observations for ALMA are calculated using the Common Astronomy Software Application v4.5 (CASA; McMullin et al. 2007). Visibilities are calculated with the simobserve task while imaging is performed with the clean task. Since our goal is not to fit the observations exactly, but merely to show the morphological similarities between our models and the observations, we do not use exactly the same $(u, v)$ coordinates as the observations to calculate our visibilities. Instead, we choose an antenna configuration in CASA (alma.out13), which results in a very similar synthesized beam to that of the real data: 0 ". $27 \times 0$ "' 25 with PA $=86^{\circ}$. To ensure our synthetic observations are as close to the real data as possible, we use a bandwidth of $6.8 \mathrm{GHz}$, assume a precipitable water vapor column of $2.7 \mathrm{~mm}$, and a total integration time of $725 \mathrm{~s}$.

1 http://www.ita.uni-heidelberg.de/ dullemond/software/radmc-3d/ 


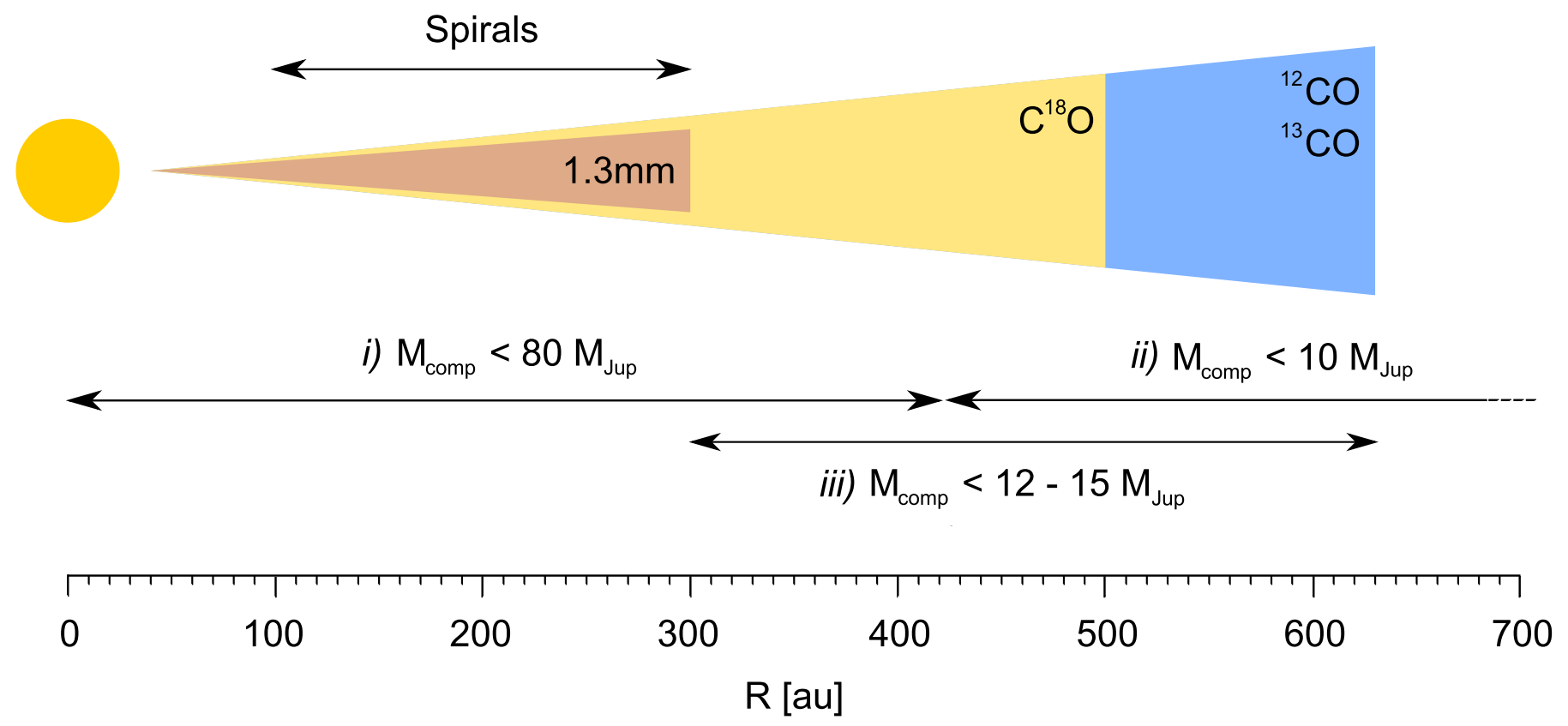

Figure 2. Schematic diagram of the Elias 2-27 system based on the results of Pérez et al. (2016). Also shown are the companion mass limits based on observations (i) by Ratzka et al. (2005), (ii) from the UKIDSS data, and (iii) theoretical gap-opening limits (see Section 2.3.2).

We then apply an unsharp masking filter to the images in a similar manner to Pérez et al. (2016). This involves convolving the image with a Gaussian kernel with a full width half maximum (FWHM) of 0 !' 33 and subtracting a scaled version of the result from the original image. This acts to remove largescale emission and boost the contrast of small-scale structures.

\subsection{Observational Constraints}

We describe various observational constraints that apply to the Elias 2-27 system, which we use when testing the three possible scenarios.

\subsubsection{Constraints on the Total System Mass}

The ${ }^{12} \mathrm{CO} J=2-1$ emission toward Elias 2-27 seems to be undergoing Keplerian rotation out to $\sim 630 \mathrm{au}$, and according to kinematic modeling, the enclosed mass interior to the emission is $0.5 \pm 0.2 M_{\odot}$ (Pérez et al. 2016). However, absorption by the surrounding molecular cloud significantly obscures the redshifted component of the emission, leading to some uncertainty in these derived masses.

\subsubsection{Constraints on the Mass of a Potential Companion}

A volume-limited multiplicity survey of the $\rho$-Ophiuci molecular cloud was performed by Ratzka et al. (2005). Elias 2-27 was found to be a single star with an upper limit on the K-band contrast of $2.5 \mathrm{mag}$ between 0 ". 13 and 6!" 4 . Additionally, the area around Elias 2-27 has been targeted by the UKIRT Infrared Deep Sky Survey (UKIDSS; e.g., Lawrence et al. 2007). The closest point source is located approximately $14^{\prime \prime}$ to the south $\left(16^{\mathrm{h}} 26^{\mathrm{m}} 44^{\mathrm{s}} 95,-24^{\circ} 23^{\prime} 21.88^{\prime \prime}\right)$ with a K-band magnitude of 15.9 mag, giving a K-band contrast of 7.5 mag with Elias 2-27. The limiting magnitude of the survey in the K-band (17.8 mag) suggests a maximum contrast with Elias 2-27 of 9.4 mag for any undetected sources.

Using the NextGen atmosphere models (Allard et al. 1997; Baraffe et al. 1998; Hauschildt et al. 1999), we convert these
K-band contrasts to upper limits for the mass of any potential companion, $M_{\text {comp }}$, for various orbital distances from Elias 2-27. Assuming all objects lie at the same distance as Elias 2-27 (139 pc), the UKIDSS data exclude any unseen companions $>0.01 M_{\odot}$ beyond 420 au (with the exception of the closest source mentioned above, which would translate to $M_{\text {comp }} \approx 0.02 M_{\odot}$ at $2000 \mathrm{au}$ ). Inside 420 au a companion up to $0.08 M_{\odot}$ could be present based on Ratzka et al. (2005).

Pérez et al. (2016) present ${ }^{12} \mathrm{CO}, \mathrm{C}^{18} \mathrm{O}$, and ${ }^{13} \mathrm{CO}$ observations, which combined show the presence of gas out to $\approx 630$ au. Based on their channel maps and the PV diagram of ${ }^{12} \mathrm{CO}$, there is no strong indication of a gap in the gas (though we note that the signal-to-noise is low). In order to not open up a gap in the gas, a planet must satisfy the viscosity and pressure conditions for gap-opening (Equations (68) and (69) of Lin \& Papaloizou 1993; see also Crida et al. 2006). Between 300 and 420 au (i.e., from the spirals to where the UKIDSS observations become relevant), the pressure condition is more stringent for $\alpha \lesssim 0.015$ providing a gap-opening mass of $\approx 13 M_{\text {Jup }}$ (though this mass limit is likely to be higher for migrating planets; Malik et al. 2015). Beyond 420 au the UKIDSS limit is more stringent than any realistic gap-opening mass. Figure 2 shows a schematic diagram illustrating the observational and theoretical constraints.

\section{Results}

We require the unsharp masked synthetic observations of the simulated disks to display morphology that is consistent with the observations. This constitutes three main features-(i) two large-scale symmetric spiral arms, (ii) two dark crescents interior to the spirals, and (iii) a bright inner ellipse along the major axis of the disk (see Figure 1, left). The spiral arms are visible in the unsharp masking and deprojected images while the dark crescents and bright inner ellipse are only present in the unsharp masking image. The disks presented in Figure 3 employ a steep outer disk edge rather than an exponentially tapered disk. 

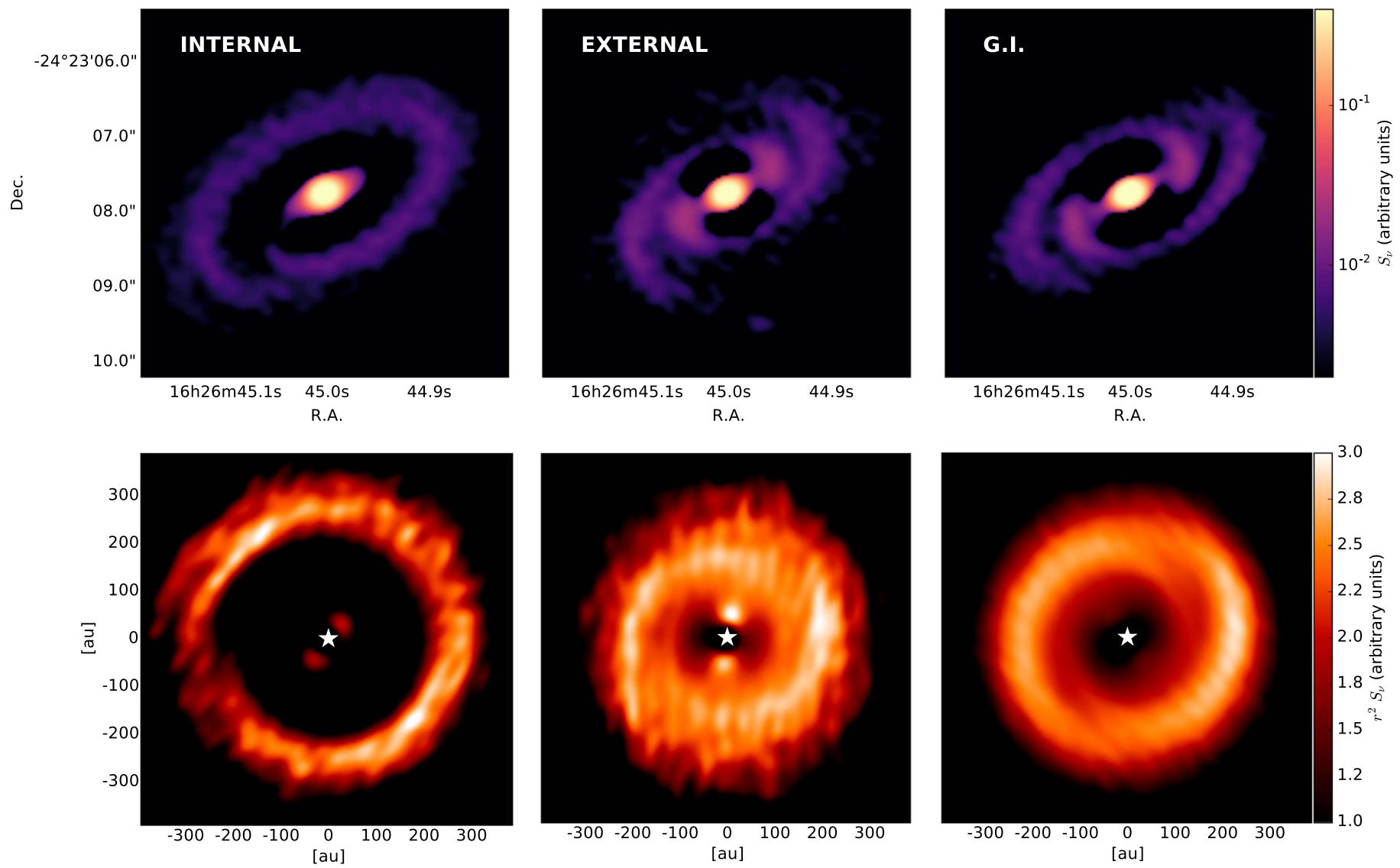

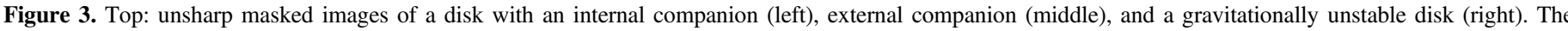

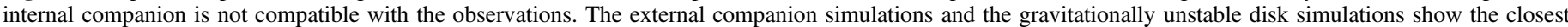

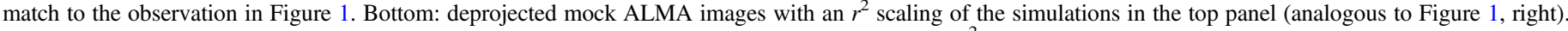

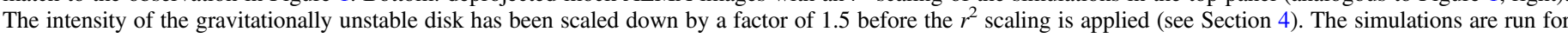
$1.6,1.2$, and 3.75 orbits at 350 au in the internal companion, external companion, and self-gravitating disk simulations, respectively.

\subsection{Internal Companion}

Figure 3 (left) shows the simulated observations for one of our internal companion simulations. The disk is a $0.08 M_{\odot}$ disk with $\Sigma \propto R^{-0.75}$ and $T \propto R^{-0.75}$, and includes a $0.01 M_{\odot}$ companion at 140 au that is allowed to accrete from the disk and grow to approximately $0.03 M_{\odot}$. The companion clears a large gap in the disk, forming the required central elliptical feature, but without the dark crescents or two-armed spiral as in the original unsharp masking observations. A lower companion mass does not generate the large-scale spirals, while highermass companions remove large amounts of material from the disk. We therefore suggest that the morphology in Elias 2-27 is unlikely to be due to an undetected companion internal to the spirals.

\subsection{External Companion}

Figure 3 (center) shows the simulated observations for one of our external companion simulations. This disk is the same as that in Section 3.1 but includes a $\approx 10 M_{\text {Jup }}$ companion at $\approx 425 \mathrm{au}$. The simulated unsharp masking observation reproduces the large-scale spiral arms, the dark crescents and the bright inner ellipse, while the deprojected image shows two large-scale spirals analogous to Figure 1 (right). We also note that simulations with companions located much beyond the gas

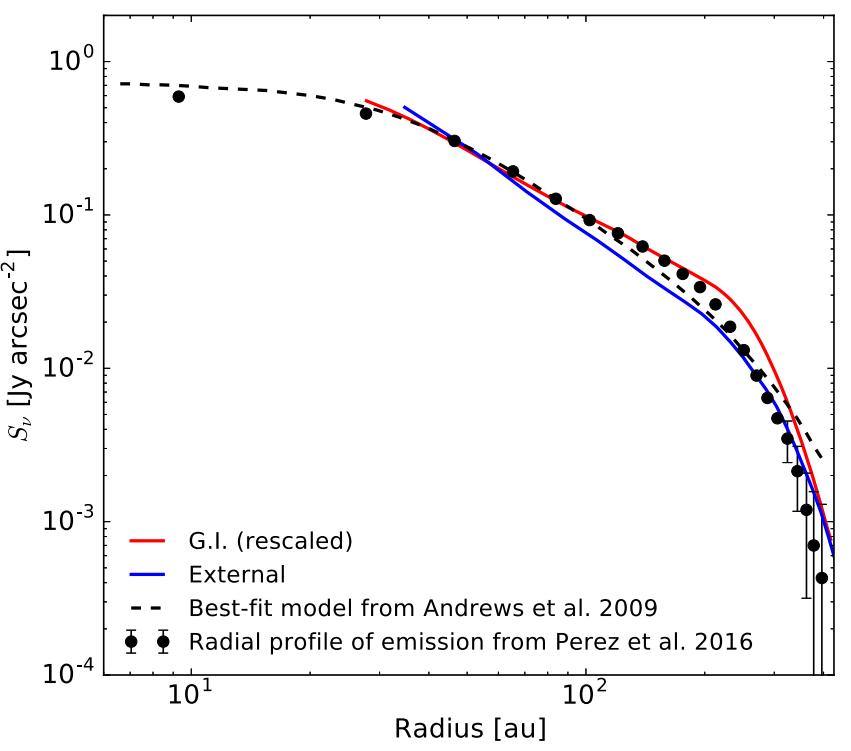

Figure 4. Radial emission profiles for the successful gravitationally unstable disk simulation (red), which has been decreased by a factor of $\sim 1.5$ (see the text) and the successful companion disk simulation (blue). Also shown are data from Pérez et al. (2016; black points) and the best-fit model from Andrews et al. (2009; black dashes). The steep decline beyond $\approx 300$ au is key to matching the observed morphology in the simulated images. 

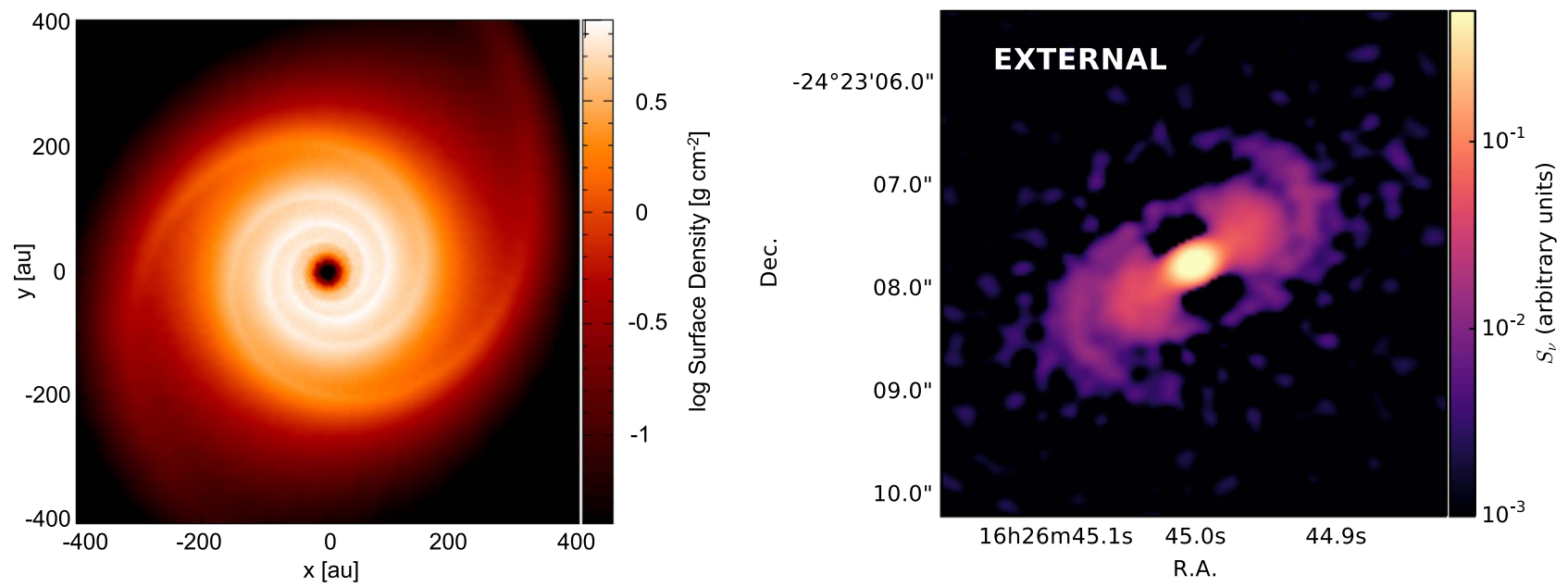

Figure 5. Left: surface density of an external companion simulation (see Section 4 for details) that displays prominent two-armed spirals. This simulation involves a $0.5 M_{\odot}$ companion on a circular orbit at $R=1200$ au and is run for 1.3 orbits at 1200 au. Right: unsharp masked image of this simulation, which is unable to reproduce the morphology due to higher levels of emission in the inner disk.

disk ( $\gtrsim 700 \mathrm{au}$ ) can only reproduce the observed morphology by violating the companion mass limits in Section 2.3.2.

\subsection{Gravitationally Unstable Disk}

Figure 3 (right) shows the simulated observations for a gravitationally unstable disk with $\Sigma \propto R^{-0.5}$ and $T \propto R^{-0.75}$. The disk mass is $0.24 M_{\odot}$, leading to a disk-to-star-mass ratio of 0.49 . Even with this relatively massive disk, the combined system mass lies within the limits discussed in Section 2.3.1. The simulated unsharp masking observation reproduces the large-scale spiral arms, the dark crescents, and the bright inner ellipse, while the deprojected image shows two large-scale spirals analogous to Figure 1 (right).

\section{Discussion}

The combination of our hydrodynamic modeling and simulated observations allows us to put strong constraints on the disk structure from which the submillimeter continuum emission originates. Throughout all of our models, we find that a steeply declining surface mass density beyond $\approx 300$ au is key to producing a close match to the masked image. Figure 4 shows the scaled radial intensity profile of our successful selfgravitating disk and the radial intensity profile of the successful companion simulations compared to the observational data from Pérez et al. (2016) and the model of Andrews et al. (2009). The steep decline beyond $\approx 300$ au in our simulation manifests itself in the form of a steep emission profile at large radii that turns over at approximately the right radius, matching the observed profile well. The modest scaling factor for the self-gravitating disk $(\sim 1.5)$ is well within uncertainties associated with dust-to-gas ratios and/or grain opacities for circumstellar disks.

We note that the resulting Toomre profile dips marginally below 1 for a limited radial range in our successful selfgravitating disk. However, this disk shows no sign of fragmentation even though we evolve it for many dynamical times for this radial range. On the other hand, a companion beyond the spirals ( $\gtrsim 300 \mathrm{au}$ ) is unlikely to have formed by core accretion. This suggests that, should the companion hypothesis be correct, this would hint that the Elias 2-27 disk may have formed a fragment by gravitational instability in the past.

We also stress the importance of applying an unsharp mask to our simulated observations. While such a mask is primarily applied to increase the contrast of the image, subtle changes are introduced to the resulting image that allow us to remove models from consideration. Changes in the surface density profile affect the radial locations at which there is an excess or deficit with respect to a Gaussian mask. This is illustrated in Figure 5, which shows a disk modeled using the properties presented by Pérez et al. (2016; see Section 2.1 for details), i.e., with an exponential surface mass density profile, which does not reproduce the observations well. Therefore, while a range of models replicate the spirals seen in the unprocessed ALMA image (e.g., Tomida et al. 2017), comparison of masked images constrains the disk properties further.

However, special care must be taken when interpreting unsharp masked images. This is because some structures may not be real, but artificially created by the masking. For example, while Figure 1 (left) superficially suggests that a gap exists in the disk, similar structures in the masked images of the external companion and self-gravitating disk in Figure 3 are generated by the interaction of the mask with a smoothly declining emissivity profile. Masked images of observations therefore need to be interpreted via the type of forward modeling exercise undertaken here.

A two-armed spiral structure, as observed around Elias 2-27, is consistent with high disk-to-star-mass ratios (e.g., Lodato \& Rice 2005). Indeed, Tomida et al. (2017), who suggested that the Elias 2-27 disk is self-gravitating, obtained a high disk-tostar-mass ratio in their simulations. The shocks associated with spiral arms in self-gravitating disks have been shown to have an effect on the chemistry of the disk material (Ilee et al. 2011; Hincelin et al. 2013; Evans et al. 2015) leading to the prospect of detecting the features in line emission (Douglas et al. 2013). Future observations of the Elias 2-27 system, with sensitivities high enough to spatially resolve relevant molecular line transitions (e.g., $\mathrm{CO}, \mathrm{HCO}^{+}$, OCS, and $\mathrm{H}_{2} \mathrm{CO}$ ) will be crucial in further evaluating the dynamics occurring within the stardisk system.

Finally, deep near-infrared imaging should offer a first step toward deciding which scenario is at work in the system. If no 
companion is detected, gravitationally instability is likely. Otherwise further follow-up observations would be required to confirm any possible detected companion.

\section{Conclusions}

We present the results of a series of hydrodynamic and radiative transfer models to test three hypotheses regarding the origin of the disk morphology around Elias 2-27-a companion internal or external to the spirals, and a gravitationally unstable disk. Our results show that a steep decline in surface mass density beyond $\approx 300$ au is required, and that the gravitational instability hypothesis or a $\lesssim 10-13 M_{\text {Jup }}$ companion between $\approx 300-700$ au can reproduce all components of the observed morphology. Given this, we suggest that Elias 2-27 may be one of the first examples of an observed self-gravitating disk or a disk that has recently fragmented forming a $\lesssim 10-13 M_{\text {Jup }}$ planet.

We thank Mike Irwin for obtaining UKIDSS photometry, and Jim Pringle and the referee for useful comments. We acknowledge support from the DISCSIM project, grant agreement 341137 under ERC-2013-ADG. F.M. acknowledges support from The Leverhulme Trust. This Letter uses the following ALMA data: ADS/JAO.ALMA\# 2013.1.00498.S. This work used the Darwin DiRAC HPC cluster at the University of Cambridge and was undertaken on the Cambridge COSMOS SMP system, part of the STFC DiRAC HPC Facility supported by BIS NeI capital grant ST/J005673/1 and STFC grants ST/H008586/1, ST/K00333X/1.

\section{References}

Allard, F., Hauschildt, P. H., Alexander, D. R., \& Starrfield, S. 1997, ARA\&A, 35, 137

ALMA Partnership, Brogan, C. L., Pérez, L. M., et al. 2015, ApJL, 808, L3 Andrews, S. M., Wilner, D. J., Hughes, A. M., Qi, C., \& Dullemond, C. P. 2009, ApJ, 700, 1502
Andrews, S. M., Wilner, D. J., Zhu, Z., et al. 2016, ApJL, 820, L40 Baraffe, I., Chabrier, G., Allard, F., \& Hauschildt, P. H. 1998, A\&A, 337, 403 Bate, M. R., Bonnell, I. A., \& Price, N. M. 1995, MNRAS, 277, 362 Benz, W. 1990, in Numerical Modelling of Nonlinear Stellar Pulsations Problems and Prospects, ed. J. R. Buchler (Dordrecht: Kluwer), 269

Birnstiel, T., Dullemond, C. P., \& Pinilla, P. 2013, A\&A, 550, L8

Booth, R. A., \& Clarke, C. J. 2016, MNRAS, 458, 2676

Casassus, S., van der Plas, G., Perez M, S., et al. 2013, Natur, 493, 191 Clarke, C. J., \& Lodato, G. 2009, MNRAS, 398, L6

Crida, A., Morbidelli, A., \& Masset, F. 2006, Icar, 181, 587

Douglas, T. A., Caselli, P., Ilee, J. D., et al. 2013, MNRAS, 433, 2064

Evans, M. G., Ilee, J. D., Boley, A. C., et al. 2015, MNRAS, 453, 1147

Evans, N. J., II, Dunham, M. M., Jørgensen, J. K., et al. 2009, ApJS, 181, 321

Hauschildt, P. H., Allard, F., \& Baron, E. 1999, ApJ, 512, 377

Hincelin, U., Wakelam, V., Commerçon, B., Hersant, F., \& Guilloteau, S. 2013, ApJ, 775, 44

Ilee, J. D., Boley, A. C., Caselli, P., et al. 2011, MNRAS, 417, 2950

Isella, A., Carpenter, J. M., \& Sargent, A. I. 2009, ApJ, 701, 260

Isella, A., Guidi, G., Testi, L., et al. 2016, PhRvL, 117, 251101

Lawrence, A., Warren, S. J., Almaini, O., et al. 2007, MNRAS, 379, 1599

Lin, D. N. C., \& Papaloizou, J. C. B. 1993, in Protostars and Planets III, ed.

E. H. Levy \& J. I. Lunine (Tucson, AZ: Univ. Arizona Press), 749

Lodato, G., \& Rice, W. K. M. 2005, MNRAS, 358, 1489

Luhman, K. L., \& Rieke, G. H. 1999, ApJ, 525, 440

Malik, M., Meru, F., Mayer, L., \& Meyer, M. 2015, ApJ, 802, 56

McMullin, J. P., Waters, B., Schiebel, D., Young, W., \& Golap, K. 2007, in ASP Conf. Ser. 376, Astronomical Data Analysis Software and Systems XVI, ed. R. A. Shaw, F. Hill, \& D. J. Bell (San Francisco, CA: ASP), 127 Meru, F. 2015, MNRAS, 454, 2529

Monaghan, J. J. 1992, ARA\&A, 30, 543

Morris, J. P., \& Monaghan, J. J. 1997, JCoPh, 136, 41

Natta, A., Testi, L., \& Randich, S. 2006, A\&A, 452, 245

Paardekooper, S.-J., \& Mellema, G. 2006, A\&A, 453, 1129

Pérez, L. M., Carpenter, J. M., Andrews, S. M., et al. 2016, Sci, 353, 1519

Price, D. J., \& Bate, M. R. 2007, MNRAS, 377, 77

Ratzka, T., Köhler, R., \& Leinert, C. 2005, A\&A, 437, 611

Ricci, L., Testi, L., Natta, A., \& Brooks, K. J. 2010, A\&A, 521, A66

Shi, J.-M., \& Chiang, E. 2014, ApJ, 789, 34

Tobin, J. J., Kratter, K. M., Persson, M. V., et al. 2016, Natur, 538, 483

Tomida, K., Machida, M. N., Hosokawa, T., Sakurai, Y., \& Lin, C. H. 2017, ApJL, 835, L11

Weingartner, J. C., \& Draine, B. T. 2001, ApJ, 548, 296

Whitehouse, S. C., \& Bate, M. R. 2006, MNRAS, 367, 32

Whitehouse, S. C., Bate, M. R., \& Monaghan, J. J. 2005, MNRAS, 364, 1367

Zhu, Z., Nelson, R. P., Dong, R., Espaillat, C., \& Hartmann, L. 2012, ApJ, 755,6 